\title{
Deep Impact and sample return
}

\author{
Michael F. A'Hearn ${ }^{1}$, Michael J. S. Belton², Steven M. Collins ${ }^{3}$, Tony L. Farnham ${ }^{1}$, Lori M. Feaga ${ }^{1}$, \\ Olivier Groussin ${ }^{1}$, Carey M. Lisse ${ }^{4}$, Karen J. Meech ${ }^{5}$, Peter H. Schultz ${ }^{6}$, and Jessica M. Sunshine ${ }^{1}$ \\ ${ }^{1}$ Dept. of Astronomy, University of Maryland, College Park, MD 20742-2421, USA \\ ${ }^{2}$ BSEI, 430 S. Randolph Way, Tucson, AZ 85716, USA \\ ${ }^{3}$ Jet Propulsion Laboratory, 4800 Oak Grove Dr., Pasadena, CA 91109, USA \\ ${ }^{4}$ JHU Applied Physics Laboratory, SD/SRE, ZMP-3, 7707 Montpelier Rd W-155, Laurel, MD 20723, USA \\ ${ }^{5}$ Inst. for Astronomy, 2680 Woodlawn Dr., Honolulu, HI 96822, USA \\ ${ }^{6}$ Geological Sciences, Brown University, Providence, RI 02912, USA
}

(Received September 12, 2006; Revised October 1, 2007; Accepted October 4, 2007; Online published February 12, 2008)

Returning a cold sample containing the ices from a cometary nucleus has long been an unachievable goal of cometary scientists. The results from the Deep Impact encounter with comet Tempel 1 suggest that the task is much easier than previously thought. Thus a cold sample return with ice becomes an achievable goal, at least from comet Tempel 1 and plausibly from other, active Jupiter-family comets.

Key words: Comets, sample return, ice, nucleus.

\section{Introduction}

Recent and current missions to comets and asteroids are bringing a wealth of new data to invigorate this field of study. These missions include flybys (DS1, Stardust, Deep Impact), orbiters (Hayabusa, Dawn, Rosetta), Impactors (Hayabusa, Deep Impact), sample return to Earth (Stardust, Hayabusa), and landers (Rosetta). While all orbiters indirectly sample some interior properties, such as density and its variations, only Deep Impact and Rosetta sample the interior composition. For many years, cometary scientists have wanted to sample the interior of a cometary nucleus, reaching deep enough to collect and remaining cold enough to return to Earth a sample of the ices in the nucleus. Because of the large uncertainty in cometary properties, such a mission has generally been found to be too complex to be affordable. As a consequence, the only sample return missions flown thus far, Stardust and Hayabusa, have sampled dust from the coma of comet Wild 2 (presumably lifted from the surface by hydrodynamic drag) and pulverized surface material from Itokawa.

The results from Deep Impact have placed major constraints on the physical properties and the variation in composition of comet 9P/Tempel 1 and, by analogy, presumably with many other, active Jupiter-family comets. This leads us to suggest that the next generation of sample return missions after Hayabusa and Stardust should consider returning a cold sample, with ices, from a cometary nucleus.

The primary factors that have limited our ability to design a mission to sample the ices have been the lack of knowledge about the strength of the material near the surface of a cometary nucleus and a similar lack of knowledge about the depth at which the ices would be found. Other param-

Copyright (c) The Society of Geomagnetism and Earth, Planetary and Space Sciences (SGEPSS); The Seismological Society of Japan; The Volcanological Society of Japan; The Geodetic Society of Japan; The Japanese Society for Planetary Sciences; TERRAPUB. eters, such as the density of the material and the strength of the gravitational field of the nucleus, have also been unknown and are important for the detailed engineering of a mission, but they are not quite as critical for conceptually designing a mission. Estimates of the strength of the material have ranged from totally strengthless to the strength of competent rock, usually with a dependence on spatial scale. This remarkably wide range of strength impacts both the ability to penetrate the material for sampling and the ability to hold the spacecraft on the surface against any forces, such as the act of sampling itself, that would tend to lift the spacecraft from the surface. Similarly, models of the evolution of the surface layers of cometary nuclei have predicted a very wide range of depths to the volatiles, ranging from $<1 \mathrm{~m}$ to many tens or even hundreds of meters.

Here we consider the relevant results from Deep Impact's encounter with comet 9P/Tempel 1. For each result we also consider the applicability to other comets than Tempel 1 to the extent possible. We conclude with a very brief assessment of mission possibilities.

\section{Results from Deep Impact}

Key preliminary results from Deep Impact were presented by A'Hearn et al. (2005), and by Sunshine et al. (2006) and several of the faster remote sensing papers were also presented in Science. Numerous detailed results, both from in situ measurements and from remote sensing were presented in a special issue of Icarus (vol 187, \#1) that was dedicated entirely to the results from Deep Impact and in a supplemental, partial special issue (vol 190,\#2). Additional results are in press and/or have been published elsewhere.

\subsection{Strength of material}

A key result from Deep Impact is that the ejecta cone never appeared to detach from the surface. This implies an upper limit to the strength, although the exact value of that upper limit is uncertain. A'Hearn et al. (2005) reported 
the first estimate of the upper limit on the post-shock tensile strength, which was explored further by Richardson and Melosh (2006), concluding that the upper limit on the dynamic, post-shock tensile strength was roughly $200 \mathrm{~Pa}$. Holsapple and Housen (2007) have presented updated, empirical scaling laws from their own laboratory experiments to determine the pre-shock static tensile strength and have argued that the upper limit can not be pushed that low and is more like $12 \mathrm{kPa}$ and might be as high as $65 \mathrm{kPa}$. Richardson et al. (2007) have subsequently modeled the flow of the ejecta more carefully, albeit still under ballistic conditions, and deduced that the upper limit on the pre-shock tensile strength is between 1 and $10 \mathrm{kPa}$.

The highest upper limit cited, $65 \mathrm{kPa}$, is consistent with the strength of highly fractured ice, while the lowest upper limit is orders of magnitude weaker. The actual static strength could, of course, be substantially lower than any of the estimates since these are all upper limits. We note that the overhangs seen on Wild 2 by the Stardust mission require strengths of order $100 \mathrm{~Pa}$ to withstand the tiny gravity of a cometary nucleus, but no such overhangs were seen on comet Tempel 1. Thus there is no evidence for significant strength and we can firmly conclude that the material's tensile strength is less than that of block ice and much less than that of competent rock.

Noting that in geological contexts the strength of materials is typically observed to decrease with increasing spatial scale, it is important to realize that the strength for which an upper limit can be determined applies on spatial scales from the size of the impactor, $1 \mathrm{~m}$, to the size of the final crater, 10 s of $\mathrm{m}$.

2.1.1 Wider applicability Other estimates of strengths for comets, at a variety of spatial scales, have been discussed by Weissman, Asphaug, and Lowry (2005) and this section just summarizes some key points from their discussion. We know from the observations of the fragments of comet D/Shoemaker-Levy 9 and various models for the tidal stresses on the nucleus that the material of that nucleus was very weak $(\leq 1000 \mathrm{~Pa}$ and probably $\leq 10 \mathrm{~Pa}$ ) on scales of a $\mathrm{km}$ or less, the size of a typical fragment. Simulations of reaccretion into the $1-\mathrm{km}$ fragments suggest that the low strength applies also at spatial scales an order of magnitude smaller. Similarly, the disruption of sun-grazing comets requires that they be very weak.

Spontaneous breakup of comets, in the absence of tidal stresses, is also not uncommon, whether complete dissipation as in the unusual case of D/LINEAR (D/1999 S4), or into several discrete fragments as in the more common case of 73P/Schwassmann-Wachmann 3. Since the mechanism is not known, there is no quantitative way of estimating the strength, except by assuming various possible mechanisms. These analyses all require that the strength of the nucleus be very low at a wide variety of spatial scales.

We conclude therefore that our upper limits on the strength are likely valid for all comets and that cometary nuclei are generally very weak at all spatial scales.

\subsection{Density of the nucleus}

In lookback images, taken 45 to 75 minutes after the impact and well after the flyby spacecraft had passed the nucleus, one can see ejecta falling back onto the surface.
Richardson et al. (2007) have also modeled this fallback, which leads directly to a determination of the local gravity. When combined with the shape model for the nucleus (Thomas et al., 2007), this yields a bulk density for the nucleus of $0.4 \mathrm{~g} \mathrm{~cm}^{-3}$. As with the estimate of strength, ballistic motion is assumed. Hydrodynamic drag, which has been excluded from the model, leads to rather large error bars, but the density estimates are consistent with models based on non-gravitational acceleration that yield $0.45 \mathrm{~g} \mathrm{~cm}^{-3}$ (Davidsson et al., 2007). The hydrodynamic drag comes from two sources - the $10^{4}$ tons of ice (see below) that sublime to water inside the plume and the ambient coma around the impact site. The former will lead to lateral acceleration if the sublimation is primarily in the cone interior to the conical wall, thus leading to an overestimate of $\mathrm{g}$, but isotropic expansion if the ice is primarily in the wall itself, a situation for which the effect on determining $g$ is less easily estimated. The latter depends critically on the distribution of ambient outgassing around the nucleus, something that is not yet well understood (Feaga et al., 2007), but this outgassing should generally lead to an understimate of $\mathrm{g}$. The agreement of the density determined from the ballistic model for the ejecta with the solution from nongravitational accelerations suggest that the hydrodynamic drag does not significantly affect the result.

2.2.1 Wider applicability There are numerous estimates of nuclear density from models of the nongravitational orbital acceleration of cometary nuclei, a topic also reviewed in some detail by Weissman et al. (2005). These models, which are somewhat more model dependent than our approach, yield densities $<1.0$ for essentially all comets for which sufficient data exist. Thus there is no reason to consider our measured density, $0.4 \mathrm{~g} \mathrm{~cm}^{-3}$, as anything other than typical.

\subsection{Material texture}

Although the overall strength is very low, these values do not address the question of the small-scale variations in strength, i.e. the texture. Would a drilling experiment run into isolated solid rocks that could lead to sampling problems? We argue that boulders as large as a few meters are not present anywhere in our excavated area since boulders that large should appear as bright, point sources within the ejecta at our spatial resolution. We also know that the size distribution of the ejecta is different from that of the ambient outgassing. On approach, the impactor was hit by four dust particles that were large enough to be detected by, and corrected for by, the Attitude Determination and Control System (ADCS). There were 3 particles between 1 and $10 \mathrm{mg}$ and 1 particle of roughly $0.5 \mathrm{~g}$. To within the uncertainty of small number statistics, these particle hits were entirely consistent with the power law for the size distribution that had been deduced from far-infrared measurements of comet Tempel 1 by IRAS as shown in Fig. 1. This power law was the basis of the predicted fluence, both for the flyby spacecraft (Lisse et al., 2005, figure 8) and for the impactor, shown here. Lisse et al. (2006, see supplementary on-line material), in order to explain their observed mid-IR to far-IR spectra of the ejecta, have shown that the size distribution of the ejecta must have been steeper than that of the ambient dust at sizes larger than a few microns, and shallower at 


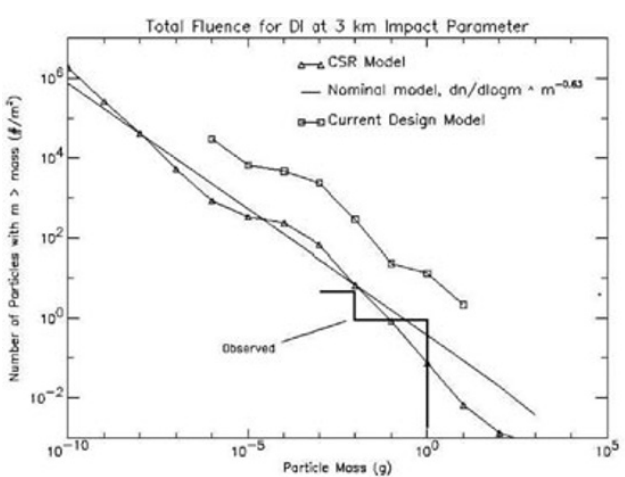

Fig. 1. Dust fluence on impactor. Predicted cumulative distribution of total dust fluence per unit area on impactor, based on the same model as shown by Lisse et al. (2005, figure 8) for the flyby spacecraft. Overlain is the histogram showing the four particles detected by the ADCS system on the impactor.

sizes smaller than about 1 micron. Simulations of the radiation pressure for several days on the ejecta do not show any need for any large particles to explain the observed structure (Schleicher et al., 2006), nor do the simulations of radiation pressure in the hour after impact (Richardson et al., 2007). However, some otherwise plausible size distributions could have a lot of mass in large particles without enough crosssection to be seen in optical imaging. Nevertheless, this argues that the larger particles that are normally present in the comae of comets are relatively fragile, easily fragmented in the excavation shock, and likely not a hazard to sampling at small scales.

We also note that the thermal measurements discussed below, which imply negligible thermal inertia across most of the nuclear surface, are not easily explained unless solid rocks larger than a few $\mathrm{cm}$ are rare, since they would provide measurable thermal inertia if they were common. Placing quantitative limits on the presence of larger rocks is beyond the scope of this paper and is one of the many tasks for continued analysis of the data from Deep Impact.

2.3.1 Wider applicability The pre-impact release of dust by comet Tempel 1 appears to be very typical of comets, at least in so far as that can be assessed from remote sensing, but we know very little about the structure of dust from other comets. The key other result that bears on this question is the clustering of impact hits on the Dust Flux Monitoring Instrument (Tuzzolino et al., 2005) on the Stardust spacecraft. One of the best interpretations of this result is that the dust is composed of weakly bonded aggregates, many of which spontaneously fragment. This is consistent with the results from DI.

\subsection{Depth of $\mathrm{H}_{2} \mathrm{O}$ ice}

We note first that Deep Impact did detect ice on the surface of the nucleus (Sunshine et al., 2006). The ice detected by Sunshine et al. covers only a very small fraction $(3 \%)$ of a small area $\left(0.5 \mathrm{~km}^{2}\right)$ and is therefore unrelated to the bulk of the outgassing by the comet. Given the location of the ice on the surface, it seems to us likely that this ice is a byproduct of the process that leads to outbursts on this portion of the nucleus (A'Hearn et al., 2005). Others have taken an opposite view, arguing that the surface ice is the

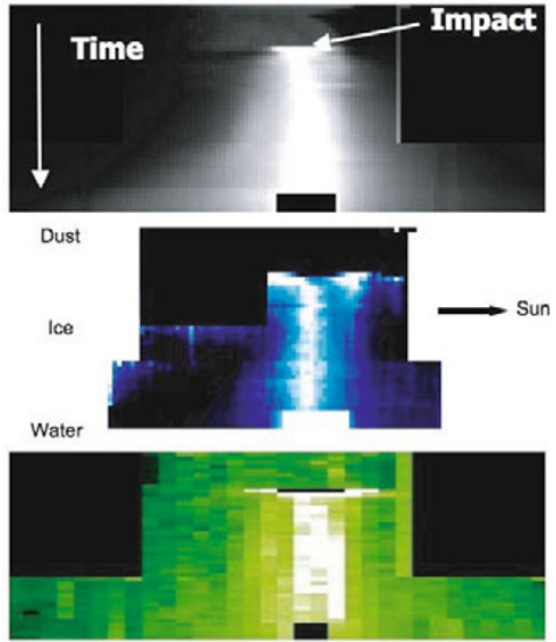

Fig. 2. Spatio-Temporal plots of ejecta. Each row is an image of the slit as ejecta flow in front of the slit. Around the time of impact, each exposure was $0.7 \mathrm{sec}$, but later (lower in each picture) the readout was increased to a longer slit and the exposure time increased to 1.4 and then $2.8 \mathrm{sec}$. Top is dust at $2 \mu \mathrm{m}$. Middle is depth of ice absorption and bottom is gaseous $\mathrm{H}_{2} \mathrm{O}$ emission. The first spectrum after impact is saturated over many pixels but ice is clearly present by the third spectrum, 2 sec after impact. Adapted from Sunshine et al. (2007).

source of the outbursts. In either case, ice is accessible at the surface and thus readily collectible. Even if the surface ice is re-deposited frost, which it may or may not be, it is of great interest for understanding the physics of comets if not their primordial structure.

Elsewhere on the nucleus, the ice is clearly not present, or at least it exhibits less than $1 \%$ surface coverage per $120 \mathrm{~m}$ pixel or it would have been seen. The ice that drives most cometary activity is below the surface and the important question is how deep that ice is, both the water ice and the more volatile ices. This will be addressed with a variety of evidence.

Spectra of the downrange ejecta immediately after impact show that ice grains, typically a few microns in size, are present in the downrange ejecta within 2 seconds of impact (Sunshine et al., 2007). Figure 2 shows a temporal sequence of 1-D images along the slit of the spectrometer as the downrange ejecta flowed across the field of view (FOV). The upper panel is a sequence at a wavelength of $\approx 2.2 \mu \mathrm{m}$, which corresponds primarily to sunlight reflected by grains. The middle panel is the ratio of two wavelengths showing the strength of the absorption feature due to icy grains at a wavelength of $2.8-3.0 \mu \mathrm{m}$, while the lower panel shows the brightness at a wavelength near the peak of the emission by gaseous $\mathrm{H}_{2} \mathrm{O}$ at $2.8 \mu \mathrm{m}$. At the top of the image, the time is prior to impact and each row in the image corresponds to an 0.7-sec exposure, with essentially no dead time between spectra. Within 3 spectra $(2 \mathrm{sec})$ after impact, ice has appeared in the downrange ejecta. As discussed by Schultz et al. (2007), these early, downrange ejecta are almost entirely from the near-surface region, within a couple of impactor diameters of the surface. Thus ice must be present within the top meter or two of material.

Sunshine et al. (2007) also show spectral maps of ice obtained at a later time that show no ice in the uprange ejecta. 


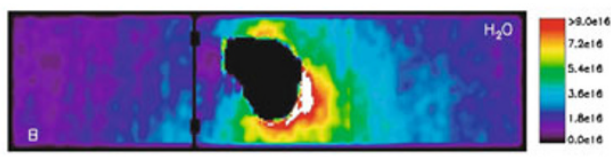

Fig. 3. Map of distribution of water. The brightness of the emission by water near the nucleus, hence the column density of water, is peaked in the areas above the hottest portions of the nucleus, which are all near the sub-solar point. The sun (ecliptic west) is to the right and ecliptic north is at the top. Adapted from Feaga et al. (2007).

Based on the experiments of Schultz et al., which imply that the uprange rays are formed from material excavated even closer to the surface than the downrange ejecta, we conclude that a very thin layer of the nucleus (few $\mathrm{cm}$ to $1 \mathrm{~m}$ ) is without ice and that a lower layer contains ice. Associating the ice with the layering in strength discussed by Schultz et al. that separates the uprange rays from the impact site seems obvious since those same rays are the ones that do not show ice.

There is a variety of other evidence that yields a similar conclusion. The first thermal map of a cometary nucleus has been described in detail by Groussin et al. (2007). The key point is that all areas where the sun is at moderate or high elevations are in local thermal equilibrium with the sunlight. This indicates that the surface temperatures (at a spatial resolution of $120 \mathrm{~m}$ ) are not reduced by significant sublimation. The upper limit on the thermal inertia $\left(50 \mathrm{~W} \mathrm{~K}^{-1} \mathrm{~m}^{-2} \mathrm{~s}^{0.5}\right.$ ) can be used, with reasonable assumptions, to determine the depth to which the thermal waves penetrate, the diurnal wave reaching approximately $10 \mathrm{~cm}$ (3 thermal skin depths) and the annual wave reaching to about $3 \mathrm{~m}$ (3 annual skin depths). These set severe constraints on interpreting the data regarding outgassing and activity.

Feaga et al. (2007) have produced the first ever spectral maps of the innermost coma of a comet, mapping within a few nuclear radii at spatial resolutions better than $1 \mathrm{~km}$. Figure 3, adapted from Feaga et al. (2007), shows the distribution of the emission by $\mathrm{H}_{2} \mathrm{O}$. Examination of this figure shows that the water appears to come predominantly from regions where the sun is at high elevations, i.e., from the subsolar region and from regions in the vicinity of the noon meridian. This implies that the water ice must be below the surface (there is no ice on the surface in this region as shown by Sunshine et al., 2006) but that it must be near enough to the surface that it is sensitive to the diurnal thermal wave. This implies that, at least in that part of the nucleus, the water ice is no deeper than 10 to $20 \mathrm{~cm}$. This sets an even tighter constraint on the depth of ice below the surface than do the cratering comparisons with laboratory work discussed above.

Kadono et al. (2007) observed in remote sensing of the ejecta that the earliest ejecta consisted of small (submicron) carbonaceous grains. From the separation of the different types of ejecta, they argue that the nucleus must have a thin layer, tens of $\mathrm{cm}$, depleted in water and that the primitive, unevolved material, including ices, is within $1 \mathrm{~m}$ of the surface. They also argue that this conclusion should be general to most Jupiter-family comets.

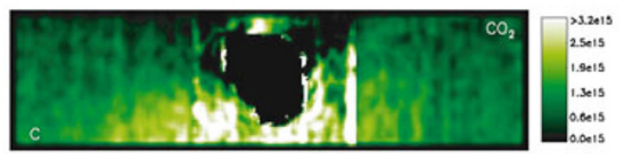

Fig. 4. Map of distribution of $\mathrm{CO}_{2}$. The brightness of the emission by $\mathrm{CO}_{2}$ near the nucleus, hence the column density of $\mathrm{CO}_{2}$, is peaked at negative latitudes (the sub-solar latitude is $+9^{\circ}$ ), including considerable emission either from the negative pole, which is in darkness, or projected in that direction but actually in the wall of the cone traced by the dust jet seen in Fig. 5. The sun (ecliptic west) is to the right and ecliptic north is at the top. Adapted from Feaga et al. (2007).

In summary, the $\mathrm{H}_{2} \mathrm{O}$ ice is very close to the surface and thus within easy reach of a variety of sampling mechanisms.

2.4.1 Wider applicability There have been in situ observations of very few comets and none have reported ice on the surface, but none of the earlier missions was well equipped to search for ice on the surface. Li et al. (2007) have argued that two areas on comet 19P/Borrelly are colder than predicted by a standard model with negligible thermal inertia and that this is potentially explained by either a small amount of ice on the surface or by cooling by the gas flow of just-sublimated surface ice. Thus there is at least one other comet with signs of ice at or very near the surface.

Some might argue that the existence of non-gravitational accelerations on cometary orbits implies a large thermal inertia or that the ice is very many skin-depths below the surface. Indeed, this lag in outgassing was the basis for Whipple's (1950) model of the icy conglomerate nucleus. However, as discussed in the recent review by Yeomans et al. (2005), asymmetries in the light curve around perihelion, the existence of jets of outgassing, significant obliquities of the nuclear rotation and other parameters all play a role in producing non-gravitational acceleration. We therefore do not view this as a strong argument against the generality of our result.

\subsection{Depth of more volatile ices}

A true understanding of cometary formation requires understanding how the various ices are mixed. Thus, a sample that returns water ice can place constraints on the formation scenario, via the crystallinity and the $\mathrm{D} / \mathrm{H}$ ratio and its variation, but a sample that includes both $\mathrm{H}_{2} \mathrm{O}$ and more volatile ices is far more valuable for understanding the mixing of species. Although $\mathrm{CO}$ is normally discussed as the dominant ice that is more volatile than $\mathrm{H}_{2} \mathrm{O}$ and the main driver of activity at large heliocentric distances, the responsivity of our spectrometer to $\mathrm{CO}$ is low. On the other hand, our data show a strong signal from $\mathrm{CO}_{2}$, a species that is intermediate in volatility between $\mathrm{H}_{2} \mathrm{O}$ and CO. Feaga et al. (2007) have deduced an abundance of $7 \%$ relative to water, comparable both to the peak of $\mathrm{CO}$ during the impact event and to the upper limit for the pre-impact ambient outgassing (Feldman et al., 2006).

Figure 4 shows that the distribution of $\mathrm{CO}_{2}$ outgassing is very different from that of the water in Fig. 3. The $\mathrm{CO}_{2}$ is being emitted primarily from the comet's negativelatitude (southern) hemisphere with the largest ratio of $\mathrm{CO}_{2}$ to $\mathrm{H}_{2} \mathrm{O}$ occuring in a direction that projects along the negative rotation axis. With the rotational pole determined from 


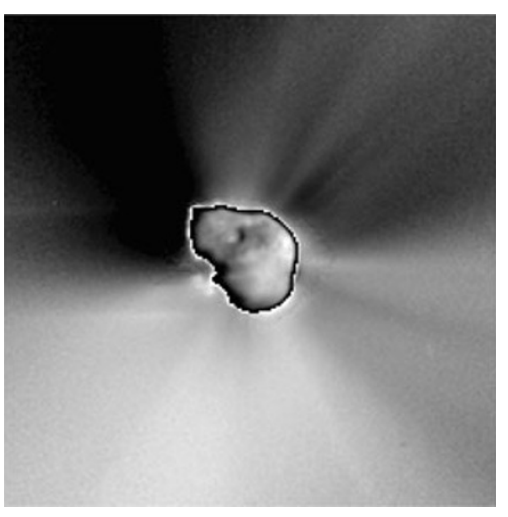

Fig. 5. Dust distribution, enhanced to show jets. One prominent jet, which is also persistently visible from Earth, traces out a cone with one edge nearly in the plane of the sky and pointing to ecliptic south and with the other edge pointing toward the observer and projecting to ecliptic southeast. The latter structure is more or less coincident with enhanced $\mathrm{CO}_{2}$ emission. From Farnham et al. (2007).

Deep Impact, the negative pole should have entered seasonal darkness ("winter") in early April, just about three months prior to the time of observation. Figure 5, from Farnham et al. (2007), is an optical (white-light) image, taken at about the same time and shown at the same spatial scale as the spectral maps. It has been enhanced to show the structure of jets, seen presumably primarily as dust. The dust jets that more or less define the lower left (ecliptic southeast) quadrant represent a classical conical structure with the south-pointing edge nearly in the plane of the sky and the southeast-pointing edge having a large component toward the observer but projecting not far from the projected negative rotational pole.

It is clear that the dust jets are not correlated with the water emission and they are much better associated with the $\mathrm{CO}_{2}$ emission, although even that correlation is far from perfect. There is an indication of night-side emission at least in areas small compared to what is visible in these images (Wellnitz et al., in preparation). Since the vernal equinox for the positive hemisphere occurred a few months earlier, we argue from these various observations that the $\mathrm{CO}_{2}$ ice is within an annual skin depth of the surface and thus probably within very roughly $1 \mathrm{~m}$ of the surface. Furthermore, $\mathrm{CO}_{2}$ was greatly enhanced in the ejecta within 2 seconds of the impact, indicating also that it must be in the uppermost meter. Whether there are either primoridial or evolutionary variations in the inherent ratio of $\mathrm{CO}_{2}$ to $\mathrm{H}_{2} \mathrm{O}$ is a question for another day.

Even super-volatiles, species much more volatile than $\mathrm{CO}_{2}$, must be within the excavated region, i.e. within the uppermost 10-20 m, since Mumma et al. (2005) observed a substantial increase in the abundance of ethane, $\mathrm{C}_{2} \mathrm{H}_{6}$, after the impact. Furthermore, the detection of post-impact $\mathrm{CO}$ by Feldman et al. (2006) in an amount comparable to the pre-impact upper limit indicates that substantial $\mathrm{CO}$ is also within the excavated region. At this point in the analysis of our own spectra, we can not set any better limits on the depth of other volatiles but further analysis should yield additional results.
2.5.1 Wider applicability We are not aware of any evidence that these results on super-volatiles are applicable to other Jupiter-family comets. The results of Biver et al. (1997) show unambiguously that highly volatile components lie within one annual skin-depth in comet Hale-Bopp, a Halley-class comet, but the applicability to Jupiter-family comets is not as clear.

\section{Discussion}

Taking the above arguments at face value, and assuming based on the limited evidence cited above that the results at Tempel 1 are typical of Jupiter-family comets, we see that ice on the surface is of very limited extent but that the bulk of $\mathrm{H}_{2} \mathrm{O}$ ice is well within $1 \mathrm{~m}$, and probably within $10 \mathrm{~cm}$, of the surface, that $\mathrm{CO}_{2}$ ice is likely within $1 \mathrm{~m}$ of the surface in selected locations, that the refractory particles in the surface layers are predominantly very weak aggregates, that there are no strong boulders larger than a few meters, and that the surface material is predominantly very weak aggregates of micron-sized grains. What are the implications for sample return missions?

It is clear that previous worries about needing to penetrate strong materials are no longer a concern. One needs to penetrate only quite weak material, even if the values by Holsapple and Housen are correct. Furthermore, penetration to a depth of 2 meters would sample not only $\mathrm{H}_{2} \mathrm{O}$ ice but also more volatile ices. The strength suggests that a sample return could be achieved by a simple coring approach while the spacecraft is held down by thrusters rather than having to anchor to the surface.

It also seems clear that any sample return mission should include a device for mapping the surface temperature at resolutions much better than $100 \mathrm{~m}$ with higher precision than was possible with Deep Impact. This is the one device that can overcome the obstacle that the ice may not be uniformly distributed below the nuclear surface.

This paper is not meant to provide engineering details, which require substantially more thought, but to indicate the feasibility of sample return missions that bring back ices from a cometary nucleus. The paper assumes that the results at this spot on the nucleus of Tempel 1 are really representative of all comets, and we have provided evidence that supports this assertion, but this is a necessary assumption at this stage of our exploration of small bodies. This can easily be tested with other impact missions and will be tested by the Rosetta mission, but we argue that planning of sample return missions need not wait for that confirmation, provided that such missions are directed to reasonably active Jupiter-family comets.

These results will be more directly tested when Philae, the lander of the Rosetta mission, measures the surface properties of comet 67P/Churyumov-Gerasimenko in 2014, but the present results are sufficient that it is not necessary to wait for that to occur before planning sample return missions.

Acknowledgments. This work was supported by the Deep Impact project, funded via NASA contract NASW00004 to the University of Maryland and via a task order to the Jet Propulsion Labortory. The authors acknowledge extensive support from the rest of the Deep Impact team. 


\section{References}

A'Hearn, M. F., et 32 al., Deep Impact: Excavating Comet Tempel 1, Science, 310, 258-264, 2005.

Biver, N., et 22 al., Long Term Evolution of the Outassing of Comet HaleBopp From Radio Observations, Earth, Moon, Planets, 78, 5-11, 1997.

Davidsson, B. J. R., P. J. Gutiérrez, and H. Rickman, Nucleus properties of Comet 9P/Tempel 1 estimated from non-gravitational force modeling, Icarus, 187, 306-320, 2007.

Farnham, T. L., D. D. Wellnitz, D. L. Hampton, J.-Y. Li, J. M. Sunshine, O. Groussin, L. A. McFadden, C. J. Crockett, M. F. A'Hearn, M. J. S. Belton, P. Schultz, and C. M. Lisse, Dust Coma Morphology in the Deep Impact Images of Comet 9P/Tempel 1, Icarus, 187, 26-40, 2007.

Feaga, L. M., M. F. A’Hearn, J. M. Sunshine, O. Groussin, and T. L. Farnham, Asymmetries in the distribution of $\mathrm{H}_{2} \mathrm{O}$ and $\mathrm{CO}_{2}$ in the inner coma of comet 9P/Tempel 1 as observed by Deep Impact, Icarus, 190, 345356, 2007.

Feldman, P. D., R. E. Lupu, S. R. McCandliss, H. A. Weaver, M. F. A'Hearn, M. J. S. Belton, and K. J. Meech, Carbon Monoxide in Comet 9P/Tempel 1 Before and After the Deep Impact Encounter, Ap. J. Letters, 647, L61-L64, 2006.

Groussin, O., M. F. A'Hearn, J.-Y. Li, P. C. Thomas, J. M. Sunshine, C. M. Lisse, K. J. Meech, T. L. Farnham, L. M. Feaga, and W. A. Delamere, Surface Temperature of the Nucleus of Comet 9P/Tempel 1, Icarus, 187, 16-25, 2007.

Holsapple, K. A. and K. R. Housen, A Crater and Its Ejecta: An Interpretation of Deep Impact, Icarus, 187, 345-356, 2007.

Kadono, T., S. Sugita, S. Sako, T. Ootsubo, M. Honda, H. Kawakita, T. Miyata, R. Furusho, and J. Watanabe, The Thickness and Formation Age of the Surface Layer on Comet 9P/Tmepel 1, Astrophys. J. Lett., 661, L89-L92, 2007.

Li, J.-Y., M. F. A'Hearn, L. A. McFadden, and M. J. S. Belton, Photometric analysis and disk-resolved thermal modeling of comet 19P/Borrelly from Deep Space 1 data, Icarus, 188, 195-211, 2007.

Lisse, C. M., M. F. A'Hearn, T. L. Farnham, O. Groussin, K. J. Meech, U. Fink, and D. G. Schleicher, The Coma of Comet 9P/Tempel 1, Space Sci. Rev., 117, 161-192, 2005.

Lisse, C. M., J. VanCleve, A. C. Adams, M. F. A’Hearn, Y. Fernández, T. L. Farnham, L. Armus, C. J. Grillmair, J. Ingalls, M. J. S. Belton, O. Groussin, L. A. McFadden, K. J. Meech, P. H. Schultz, B. C. Clark, L. M. Feaga, and J. M. Sunshine, Spitzer Spectral Observations of the Deep Impact Ejecta, Science, 313, 635-640, 2006.

Mumma, M. J., M. A. DiSanti, K. Magee-Sauer, B. P. Bonev, G. L. Villanueava, H. Kawakita, N. Dello Russo, E. L. Gibb, G. A. Blake, J. E. Lyke, R. D. Campbell, J. Aycock, A. Conrad, and G. M. Hill, Parent
Volatiles in Comet 9P/Tempel 1: Before and After Impact, Science, 310 , 270-274, 2005.

Richardson, J. E. and H. J. Melosh, Modeling the Ballistic Bahavior of Solid Ejecta from the Deep Impact Cratering Experiment, Lunar \& Plan. Sci. Conf., Abstract \# 1836, 2006.

Richardson, J. E., H. J. Melosh, C. M. Lisse, and B. Carcich, A Ballistics Analysis of the Deep Impact Ejecta Plume: Determining Comet Tempel 1's Gravity, Mass, and Density, Icarus, 190, 357-390, 2007.

Schleicher, D. G., K. L. Barnes, and N. F. Baugh, Photometry and Imaging Results for Comet 9P/Tempel 1 and Deep Impact: Gas Production Rates, Postimpact Light Curves, and Ejecta Plume Morphology, Astron. J., 131, 1130-1137, 2006.

Schultz, P. H., C. A. Eberhardy, C. M. Ernst, J. M. Sunshine, M. F A'Hearn, and C. M. Lisse, The Deep Impact Oblique Cratering Experiment, Icarus, 190, 295-333, 2007.

Sunshine, J. M., M. F. A’Hearn, O. Groussin, et 20 al., Exposed Water Ice Deposits on the Surface of Comet 9P/Tempel 1, Science, 311, 1453 1455, 2006

Sunshine, J. M., O. Groussin, P. L. Schultz, M. F. A’Hearn, L. M. Feaga, T. L. Farnham, and K. P. Klaasen, The Distribution and Structure of Water Ice in the Interior of Comet Tempel 1, Icarus, 190, 284-294, 2007.

Thomas, P. C., J. Veverka, M. J. S. Belton, A. Hidy, M. F. A'Hearn, T. L. Farnham, O. Groussin, J.-Y. Li, L. A. McFadden, J. Sunshine, D. Wellnitz, C. Lisse, P. Schultz, K. J. Meech, and W. A. Delamere, The Shape, Topography, and Geology of Tempel 1 from Deep Impact Observations, Icarus, 187, 4-15, 2007.

Tuzzolino, A. J., T. E. Economou, B. C. Clark, P. Tsou, D. E. Brownlee, S. F. Green, J. A. M. McDonnell, N. McBride, and M. T. S. H. Colwell, Dust Measurements in the Coma of Comet $81 \mathrm{P} /$ Wild 2 by the Dust Flux Monitor Instrument, Science, 304, 1776-1780, 2005.

Weissman, P. R., E. Asphaug, and S. C. Lowry, Structure and Density of Cometary Nuclei, in Comets II, edited by M. C. Festou, H. U. Keller, and H. A. Weaver, 745 pp, University of Arizona Press, Tucson, 2005.

Whipple, F. L., A comet model. I. The acceleration of Comet Encke, Astrophys. J., 111, 375-394, 1950.

Yeomans, D. K., P. W. Chodas, G. Sitarski, S. Szutowicz, and M. Królikowska, Cometary orbit determination and nongravitational force, in Comets II, edited by M. C. Festou, H. U. Keller, and H. A. Weaver, 745 pp, University of Arizona Press, Tucson, 2005.

M. F. A'Hearn (e-mail: ma@astro.umd.edu), M. J. S. Belton, S. M Collins, T. L. Farnham, L. M. Feaga, O. Groussin, C. M. Lisse, K. J. Meech, P. H. Schultz, and J. M. Sunshine 